\title{
Numerical Calculation of Secondary Current Distribution in a Two- Dimensional Electrochemical Cell with a Non-Uniform Resistive Electrode ${ }^{* 1}$
}

\author{
Hiroyuki KAWAMOTO*
}

Received August 6, 1993 ; Accepted September 24, 1993

\begin{abstract}
A new numerical method has been developed to calculate secondary current distribution in electrochemical cells. The method is applicable in arbitrary two-dimensional systems which include non-uniform and/or anisotropical resistive electrodes with nonlinear polarization kinetics such as the Butler-Volmer polarization at electrolyte-electrode interfaces. The calculation uses a double iterative method employing the boundary element method (BEM) in the electrolyte region and the finite element method (FEM) in the electrode region. Parametric calculations for a corner electrode were performed to demonstrate the present method.
\end{abstract}

\section{INTRODUCTION}

It is essential in the design of electrochemical systems to accurately calculate the distribution of current density over electrode surfaces without any geometrical or kinetic simplification1, 2). Though electrodes are usually assumed to be infinitely conductive in calculating secondary current distributions ${ }^{3 \sim 10}$ ), a potential drop in electrodes is not negligible in the case of microelectronic electrodes. The author developed a numerical method ${ }^{15)}$ to calculate secondary current distribution for arbitrary two-dimensional electrochemical cells having electrodes of finite conductivities. However, the method is still restricted to uniform resistive electrodes. Non-

\footnotetext{
Manufacturing Engineering Laboratory, Corporate Research Laboratories, FUJI XEROX Co., Ltd. (2274 Hongo, Ebina - shi, Kanagawa 243-04, Japan)

*1 This paper was presented at The $183 \mathrm{rd}$ Meeting of The Electrochemical Society, Inc., cosponsored by The Electrochemical Society of Japan and The Japan Society of Applied Physics, Honolulu, Hawaii in 1993 and published in the proceedings volume "CHLOR - ALKALI AND CHLORATE PRODUCTION AND NEW MATHEMATICAL AND COMPUTATIONAL METHOD IN ELECTROCHEMICAL ENGINEERING".

Key Words: Secondary Current Distribution, Mathematical Modeling, Boundary Element Method, Finite Element Method
}

uniformity of the electrode conductivity should be considered when the electrode is made of layered films or impurity doped materials. If the electrode is made of compounds containing fibers, anisotropic features should also be taken into consideration. For example, microelectronic circuits or resistors by means of the thick film technology11) are sometimes highly non-uniform due to non-uniform spatial distribution of metal particles and glass. Since the conduction mechanism in thick films is point-to-point contact of metal particles, small non - uniformity of the particle distribution causes large nonuniformity of the resistivity. A non-magnetic contact development roller ${ }^{12}$, a biased transfer roller ${ }^{13)}$, and a charger roller ${ }^{14)}$ of the xerographic engine are other examples of the nonuniform resistive electrode. The roller consists of a metal shaft and conductive elastic layer(s). Since the layer is made of resin or rubber containing carbon particles, these resistivity are non-uniform even in the single layer roller due to the same reason of the thick film circuit. Resistive fibers are sometimes mixed in the resin to control the resistivity. Radial resistivity is high compared with axial resistivity in this case, if the roller is made by the injection molding method. 
The purpose of the present work is to establish a method of numerical analysis to calculate secondary current distribution, which is applicable to arbitrary two-dimensional electrochemical cells having electrodes of non-uniform and/or anisotropic conductivities with nonlinear Butler-Volmer polarization kinetics.

\section{MODELING}

\subsection{Basic equations}

It is assumed that an electrochemical cell consists of a well stirred electrolyte with no concentration gradients, and electrodes, anode and cathode, whose conductivities are non - uniform and/or anisotropic. The following two equations (1) and (2) apply in the electrolyte region ${ }^{16}$ ) and the electrode region 17 , respectively.

$$
\begin{aligned}
& \nabla^{2} \Phi_{y}=0, \\
& \nabla \cdot\left(\sigma_{e} \nabla \phi_{e}\right)=0,
\end{aligned}
$$

where $\phi$ is electric potential, $\sigma$ is conductivity, and the subscripts $y$ and $e$ denote electrolyte and electrode, respectively. It should be emphasized that the conductivity of the electrode, $\sigma_{e}$, is generally a tensor ${ }^{17)}$. From the conservation law of current, the current that flows into the electrode is equal to that from the electrolyte.

$$
\boldsymbol{n} \cdot \sigma_{y} \nabla \phi_{y}=\boldsymbol{n} \cdot \sigma_{e} \nabla \phi_{e},
$$

where $\boldsymbol{n}$ is a unit normal vector perpendicular to the electrode. On the other hand, the potential at the interfaces between the electrolyte and electrodes is not continuous, but the surface overpotential, $n_{s}$, exists.

$$
\eta_{s}=\phi_{e}-\phi_{y}
$$

The Butler-Volmer equation describes the relationship between the surface overpotential and the current density at the electrodes.

$$
\begin{aligned}
i_{n} & =-\sigma_{y} n \cdot \nabla \Phi_{y} \\
& =i_{0}\left[\exp \left(\frac{\alpha_{a} F n_{s}}{R T}\right)-\exp \left(-\frac{\alpha_{c} F n_{s}}{R T}\right)\right] .
\end{aligned}
$$

Here $i_{n}$ is the current density to the electrode, $i_{0}$ is the exchange current density, $\alpha_{a}$ and $\alpha_{c}$ are anodic and cathodic transfer coefficients, $F$ is Faraday's constant, $R$ is the universal gas constant, and $T$ is absolute temperature. The applied voltage is constant at the terminals if the electrochemical cell is operated under constant voltage, $\phi_{0}$

$$
\Phi_{a}=\phi_{0}, \quad \Phi_{c}=0 .
$$

Subscripts $a$ and $c$ denote the anode and the cathode, respectively. The natural boundary condition applies on insulated boundaries or planes of symmetry.

$$
n \cdot \nabla \Phi_{y, e}=0 .
$$

To calculate the secondary current distribution in the cell, Laplace's equation (1) and equation (2) may be solved subject to the boundary conditions (6) and (7) satisfying the conservation law of current (3) and the internal boundary condition (4) and (5). The nonlinear boundary condition (5), the difference in conductivity between the electrolyte and the electrode, and the non - uniform and/or anisotropic conductivity of the electrode complicate the numerical calculation.

\subsection{Numerical method}

A double iterative numerical method was used to solve the system of equations. A flowchart of the numerical method is shown in Fig. 1. The numerical procedure is almost the same as that reported in reference 15 except that the finite element method (FEM)18) was used in the present method to calculate the potential distribution in the electrode with non-uniform and/or anisotropic resistance, because the finite element method is suitable for the calculation of non-uniform and anisotropic fields. On the other hand, since the Laplace equation applies in the electrolyte region, the boundary element method (BEM)19, 20) was used to calculate the potential distribution in the electrolyte region for the same reason as that of the uniformly resistive electrode ${ }^{15)}$. 


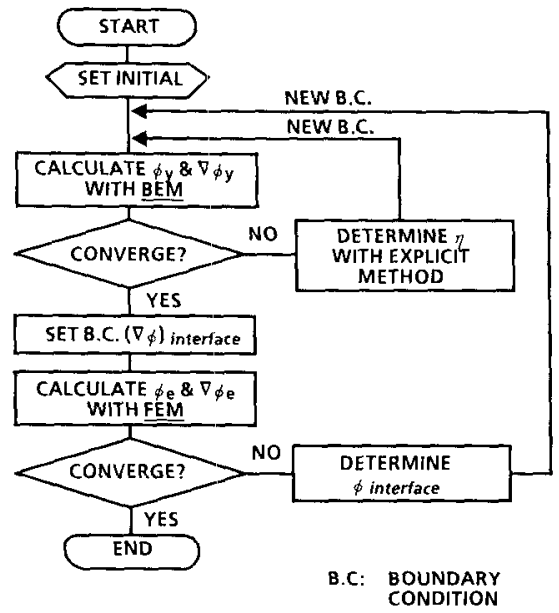

Fig. 1 Flowchart of numerical calculation based on a double iterative method using BEM and FEM.

An EPSON Model PC-386G personal computer (33 $\mathrm{MHz} 80386 \mathrm{CPU}$ with the Intel mathematical coprocessor $387 \mathrm{TMDX}-33$ ) was used with a BASIC program for numerical calculations.

\section{RESULTS AND DISCUSSION}

\subsection{Calculation conditions}

Parametric calculations for cells which consist of flat anodes and corner cathodes were performed to demonstrate the present method 15 ). Calculation parameters are listed in Table 1 and cell geometries are shown in Fig. 2. The anode was assumed to be infinitely conductive, but conductive properties of the cathode were selected as calculation parameters as well as the Wagner number. Linear basis functions were employed between the nodal points of both boundary elements and triangular finite elements.

Table 1 Calculation conditons.

\begin{tabular}{|c|c|c|}
\hline Cathode & $\begin{array}{l}W a_{L}=0 \\
\text { (Primary) }\end{array}$ & $\begin{array}{c}W a_{L}=0.5 \\
\text { (Secondary) }\end{array}$ \\
\hline Infinitely conductive & CASE 1 & CASE 2 \\
\hline $\begin{array}{l}\text { Non-uniform but } \\
\text { isotropic }\end{array}$ & CASE 3 & CASE 4 \\
\hline $\begin{array}{l}\text { Non-uniform and } \\
\text { anisotropic }\end{array}$ & CASE 5 & CASE 6 \\
\hline
\end{tabular}

a) The anode was assumed to be infinitely conductive.

b) CASEs 1,3 , and $5\left(W a_{L}=0\right)$ correspond to the primary current distribution.

c) According to Dukovic's definition 2), three dimensionless parameters which characterize the nonlinear polarization system were used in this report.

$$
\begin{aligned}
& W a_{L} \equiv R T \mathrm{o} /\left[\left(\mathrm{a}_{a}+a_{c}\right) F L i_{o}\right] ; \\
& \text { Wagner number for linear kinetics, } \\
& W a_{T} \equiv R T o /\left(a_{c} F L i_{a v e}\right) ; \\
& \text { Wagner number for Tafel kinetics, } \\
& a_{a} / a_{c}=1,
\end{aligned}
$$

where $L$ is characteristic length and $i_{\text {ave }}$ is an average current density at the interface between the cathode and the electrolyte. In the present calculation, $W a_{L}$ was given as the calculation condition but $W a_{T}$ was derived from the calculated current density at the interface between the cathode and the anode. A gap between the anode and the cathode land was selected as the characteristic length $L$ in this report.

3.2 Potential and current density distributions

Figure 3 shows the result of calculations on

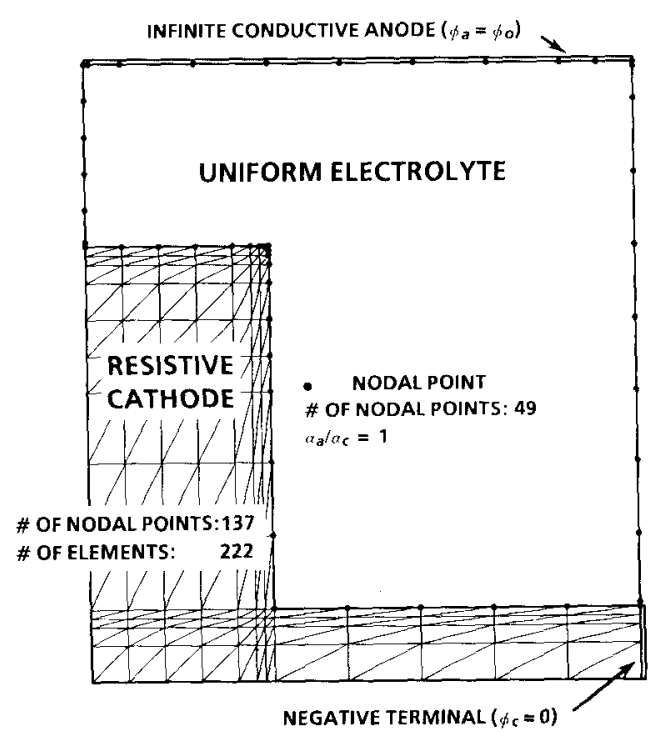

Fig. 2 Cell geometries for calculations. - nodal point, number of nodal points on electrolyte $=49$, number of nodal points in cathode $=137$, number of elements in cathode $=222$. 


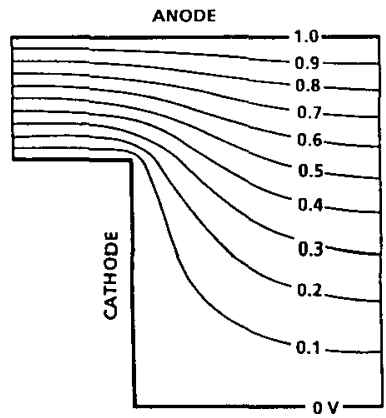

(a) CASE $1 \quad W a_{T}=0.12$

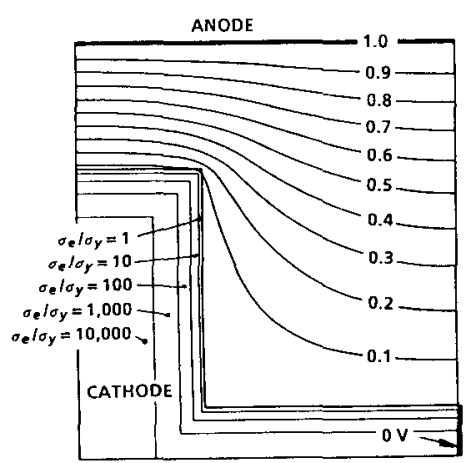

(c) CASE 3 War $=0.13$ ANODE

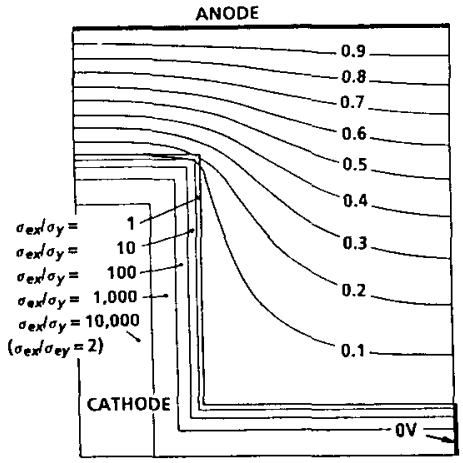

(e) CASE 5 War $=0.14$

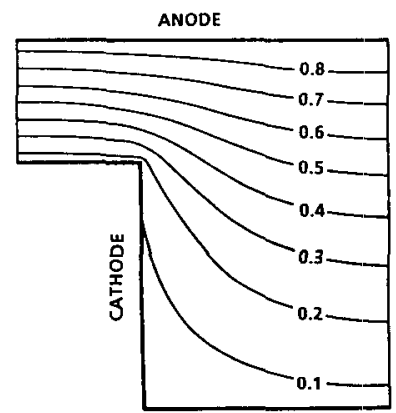

(b) CASE 2 War $=0.16$

ANODE

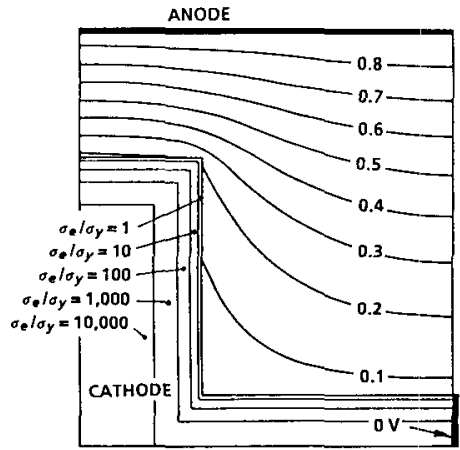

(d) CASE 4 Wa $=0.17$

ANODE

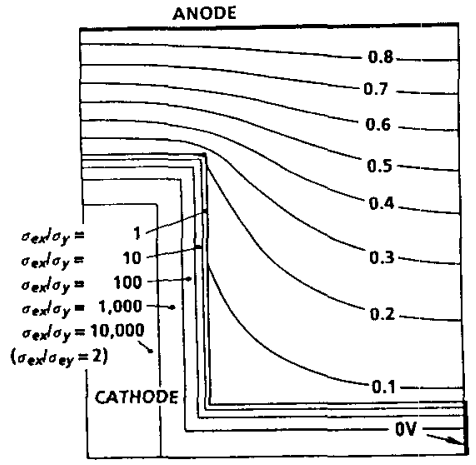

(f) CASE 6 War $=0.17$

Fig. 3 Potential distribution. Curves in the figures designate $10 \%$ pitch equi-potential surfaces.

potential distribution in the cells, and Fig. 4 is the normal current density along the cathodes. The number of iterations was around 50 , and the calculation time was about 2 hours with the compiled BASIC program for CASEs $2-6$ till convergence error, defined by $\max \left\{\left(\phi^{i \text { th }}-\phi^{i-1 \text { th }}\right) / \phi^{i \text { th }}\right\}$, becomes less than $0.01 \%$.

The following are the results from these figures.

(1) In CASE 1 the current density at the edge is theoretically infinite. On the other hand, the current density is zero in this case at the corner of the cathode. These are the typical characteristics of the primary current distribution 16,21 ).

(2) The non-polarized resistive electrode (CASEs 3 and 5) reduced current concentration 

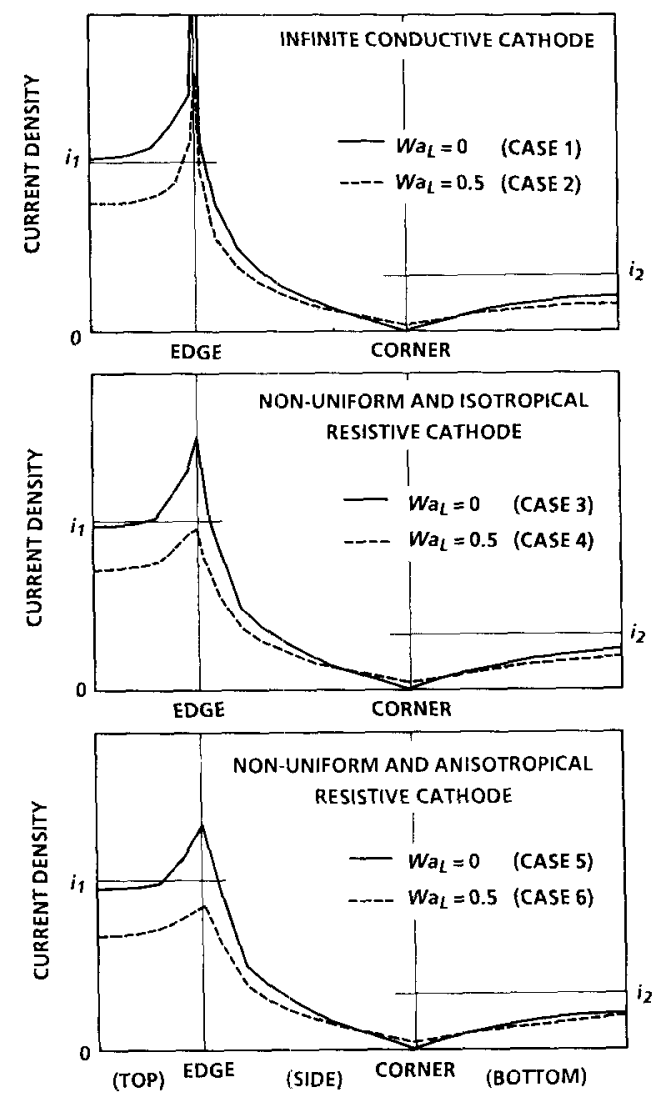

Fig. 4 Current density distributions along cathodes. The abscissa is the distance along the cathode and the ordinate is the normalized current density, where $i_{1}=\sigma \Phi_{0} / L$ (current density in case of infinite parallel plates separated at the distance of $L$ ) and $i_{2}=\sigma \Phi_{0} / L_{2}$ (current density in case of infinite parallel plates separated at the distance of $L_{2}$, where $L_{2}$ is the distance between the anode and the bottom plate of the cathode.)

at the edges. The surface overpotential did not exist at interfaces in the non - polarized cases but a potential drop due to ohmic resistance arose in the cathodes.

(3) In accordance with the increase in polarization (CASEs 2, 4, and 6), a potential drop (or surface overpotential) appeared at the electrode electrolyte interfaces and the current distribution became more uniform. The singularity at the edges was nonexistent in the polarized cases, and the current density was finite both at the edge and corner of the polarized cathodes. These are typical characteristics of secondary current distribution 16) and are qualitatively the same as those of the uniformly resistive electrode reported in reference 15 .

(4) The polarized resistive electrode (CASEs 4 and 6) further reduced current concentration at the edge because of polarization at the interfaces and the ohmic potential drop in the cathodes depending on resistive properties of the electrode.

\section{SUMMARY AND CONCLUSIONS}

A numerical method has been developed to calculate secondary current distribution in electrochemical cells. The method is applicable in arbitrary two-dimensional systems which include a uniform concentration electrolyte and non-uniform and/or anisotropical resistive electrodes with Butler-Volmer nonlinear polarization kinetics at electrolyte-electrode interfaces. The calculation uses a double iterative method employing the boundary element method in the electrolyte region and the finite element method in the electrode region coded in BASIC to obtain secondary current distribution with nonlinear polarization and finitely conductive electrodes. The present simulation method is expected to be utilized in the design of micro-electrochemical cells with non-uniform and/or anisotropical resistive electrodes.

\section{LIST OF SYMBOLS}

$F \quad$ Faraday's constant $\quad\left(96,487 \mathrm{C} \mathrm{equiv}^{-1}\right)$

$i_{\text {ave }}$ averaged current density at interface between cathode and electrolyte $\left(\mathrm{A} \mathrm{m}^{-2}\right)$

$i_{o}$ exchange current density $\left(\mathrm{A} \mathrm{m}^{-2}\right)$

$i_{n} \quad$ current density to electrode $\left(\mathrm{A} \mathrm{m}^{-2}\right)$

$L \quad$ characteristic length (m)

$n$ unit normal vector perpendicular to boundaries

$R \quad$ universal gas constant

$\left(8.3143 \mathrm{~J} \mathrm{~mol}^{-1} \mathrm{~K}^{-1}\right)$

$T \quad$ absolute temperature

$W a_{L} \quad$ Wagner number for linear kinetics

$\left(\equiv R T \sigma /\left[\left(a_{a}+a_{c}\right) F L i_{o}\right]\right)$

$W a_{T}$ Wagner number for Tafel kinetics ( $\left.\equiv R T \sigma /\left[a_{c} F L i_{a v e}\right]\right)$ 
$a_{a}, a_{c}$ anodic and cathodic transfer coefficients

$\eta_{s} \quad$ surface overpotential ( $\left.\equiv \Phi_{e}-\phi_{y}\right) \quad$ (V)

$\sigma \quad$ conductivity of electrolyte $\left(\mathrm{S} \mathrm{m}^{-1}\right)$

$\sigma_{e} \quad$ conductivity of electrode $\quad\left(\mathrm{S} \mathrm{m}^{-1}\right)$

$\Phi$ potential (V)

$\phi_{0}$ potential difference between anode and cathode terminals

(V)

\section{subscripts}

a anode

c cathode

e electrode

$y \quad$ electrolyte

\section{REFERENCES}

1) G.A. Prentice and C.W. Tobias, J. Electrochem. Soc., 129, 72 (1982).

2) J.O. Dukovic, IBM J. RES, DEVELOP., 34, 693 (1990).

3) R. Alkire, T. Bergh and R.L. Sani, J. Electrochem. Soc., 125, 1981 (1978).

4) G.A. Prentice and C.W. Tobias, J. Electrochem. Soc., 129, 78 (1982).

5) K. Tokuda, T. Gueshi, K. Aoki and H. Matsuda, J. Electrochem. Soc., 132, 2390 (1985).

6) J. Deconinch, G. Maggetto and J. Vereecken, $J$. Electrochem. Soc., 132, 2960 (1985).

7) H. Shih and H.W. Pickering, J. Electrochem. Soc., 134, 551 (1987).
8) M. Matlosz, C. Creton, C. Clerc and D. Landolt, J. Electrochem. Soc., 134, 3015 (1987).

9) F.A. Jagush, R.E. White and W.E. Ryan, J. Electrochem. Soc., 137, 1848 (1990).

10) E.C. Dimpault-Darcy and R.E. White, J. Electrochem. Soc., 135, 656 (1988).

11) C.A. Harper (edition), Handbook of Thick Film Hybrid Microelectronics, McGraw-Hill (1982).

12) M. Hosoya, M. Saito and T. Uehara, J. Imaging Science and Technology, 37, 223 (1993).

13) M.C. Zaretsky, J. Imaging Science and Technology, 37, 187 (1998).

14) H. Kawamoto, to be presented at The 9 th International Congress on Advances in NonImpact Printing Technologies/Japan Hard Copy '93, Yokohama, Japan (1993).

15) H. Kawamoto, J. Appl. Electrochem., 22, 1113 (1992).

16) J.S. Newman, Electrochemical Systems (2nd edition), Prentice-Hall, Inc. New Jersey (1991).

17) L.D. Landau and E.M. Lifshits Electrodynamics of Continuous Media, State Publishing House for Physico-Mathematical Literature, Moscow (1959)

18) O.C. Zienkiewicz, The Finite Element Method, McGraw-Hill, London (1977).

19) C.A. Brebbia, The Boundary Element Method for Engineers, Pentech Press Limited, London (1978).

20) C.A. Brebbia and S. Walker, Boundary Element Techniques in Engineering, Butterworth \& Co. Ltd., London (1980).

21) H. Kawamoto, Bulletin of the JSME, 26, 1654 (1983). 